\title{
Consistency-Enhanced Evolution for Variable Selection Can Identify Key Chemical Information from Spectroscopic Data
}

Jangwon Lee ${ }^{1}$, Jesus Flores-Cerrillo ${ }^{2}$, Jin Wang ${ }^{1 *}$, Q. Peter He ${ }^{1 *}$

${ }^{1}$ Department of Chemical Engineering, Auburn University, Auburn, AL 36849 USA

${ }^{2}$ Linde Digital, Linde plc, Tonawanda, NY, 14150

*JW: wang@auburn.edu; QPH: qhe@auburn.edu

\section{Supporting Information}

S1. Additional figures showing the effectiveness of CEEVS in extracting chemical information from NIR spectra
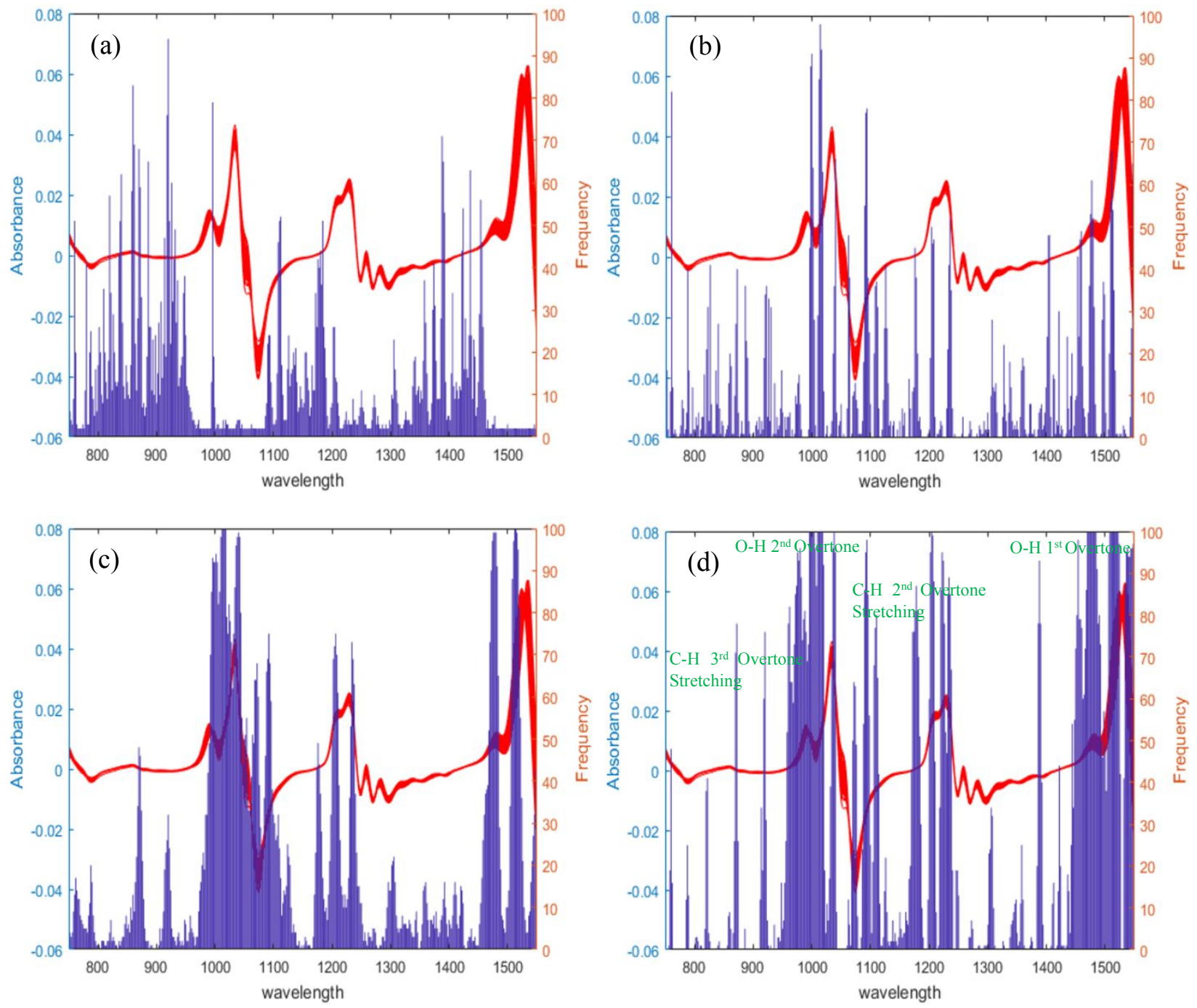

Fig. S1. Plot of spectra (red curves) and histogram of selected wavelengths (blue vertical bars) over 100 MC runs for the diesel fuel dataset. (a) CARS; (b) SVP; (c) GA; (d) CEEVS 

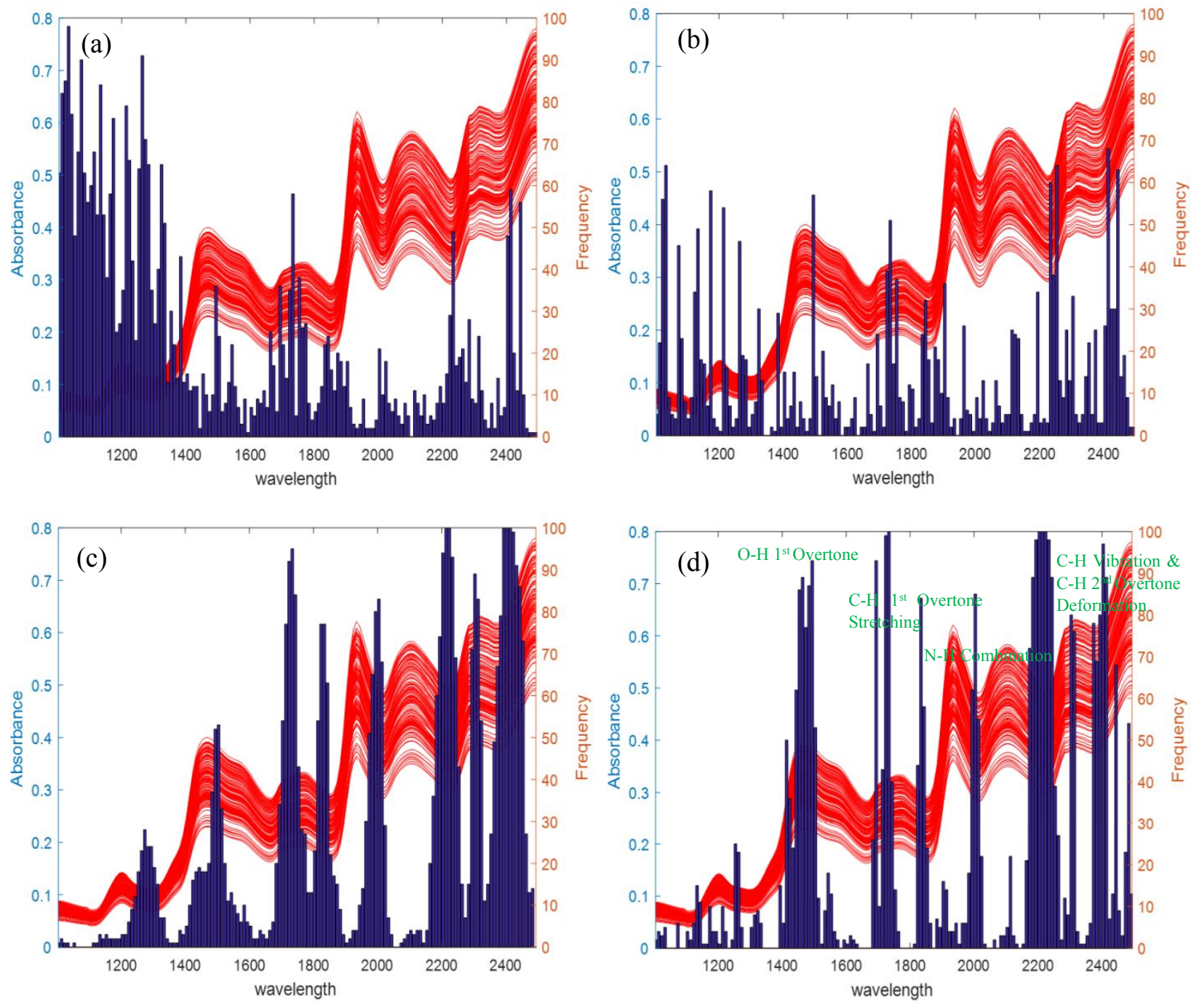

Fig. S2. Plot of spectra (red curves) and histogram of selected wavelengths (blue vertical bars) over 100 MC runs for the wheat dataset. (a) CARS; (b) SVP; (c) GA; (d) CEEVS 

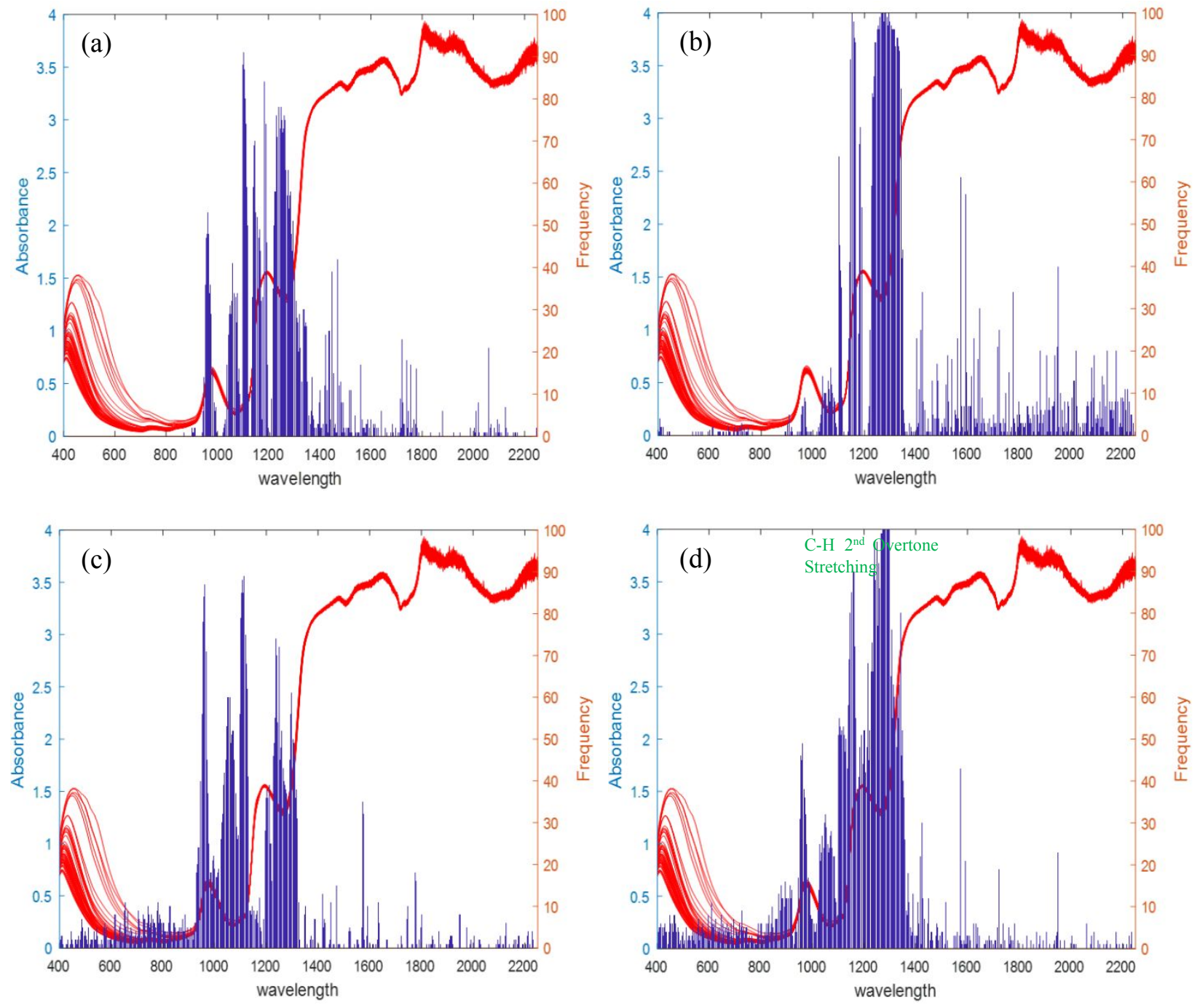

Fig. S3. Plot of spectra (red curves) and histogram of selected wavelengths (blue vertical bars) over 100 MC runs for the beer dataset. (a) CARS; (b) SVP; (c) GA; (d) CEEVS 